\title{
Endogenous Circannual Clock and HP Complex in a Hibernation Control System
}

\author{
N. KONDO \\ Mitsubishi Kagaku Institute of Life Sciences, Machida, \\ Tokyo 194-8511, Japan
}

\begin{abstract}
Hibernation in mammals is a mysterious biological phenomenon that appears on a seasonal basis for surviving a potentially lethal low body temperature $(\mathrm{Tb})$ near $0^{\circ} \mathrm{C}$ and protecting organisms from various diseases and harmful events during hibernation. The exact mechanism by which such a unique ability is seasonally developed is still unknown. On the basis of our previous finding that the source of calcium ions for excitation-contraction coupling in myocardium of chipmunks, a rodent hibernator, is seasonally modulated for hibernation, the liver-derived hibernation-specific protein (HP) complex was discovered. Recently, the HP complex was identified as a promising candidate hormone that carries a hibernation signal to the brain independently of $\mathrm{Tb}$ and environmental changes for developing a capacity for tolerating low $\mathrm{Tb}$. This finding will promote new approaches to understanding biological hibernation systems, including a circannual clock and its signaling pathway between the brain and the periphery. A new definition of hibernation and a possible model of a hibernation control system are proposed.
\end{abstract}

\section{INTRODUCTION}

Mammalian hibernation has long been of interest as a mysterious biological phenomenon. During hibernation, $\mathrm{Tb}$ decreases to about $0^{\circ} \mathrm{C}$, and then metabolic responses are remarkably depressed. Such a profoundly depressed metabolism generally leads to the dysfunction of various cells and tissues in nonhibernators, resulting in lethal damage and eventual death. However, hibernating animals survive such a severely depressed physiological state without any damage. At a decreased $\mathrm{Tb}$ during hibernation, the heart rate dramatically decreases with greatly reduced blood flow, yet the animals can tolerate these hypothermic and ischemic stresses. Thus, hibernators possess the ability to develop a tolerance to a lowtemperature disturbance of metabolism during hibernation in order to prevent cold injuries.

Hibernation has been further suggested to increase tolerances to hypoxia and hypoglycemia in the brain, bacterial infections, tumorigenesis, radiation, disuse muscle atrophy (see Kondo et al. 2006), and osteoporosis (Donahue et al. 2006). In immune functions in a hibernating state, the classical pathway of the serum complement system is active (Maniero 2002) and the splenic macrophages bind more bacterial lipopolysaccharide (Maniero 2005). Furthermore, hibernators live much longer even under warm conditions where a decreased $\mathrm{Tb}$ is prevented (Zivadinovic and Andjus 1996; Kondo and Narita 1998; Kondo 1999). Thus, hibernation is proposed to be a seasonally regulated physiological adaptation that is developed specifically for maintaining health and life by protecting organisms from severe physiological states. If better understood, hibernation would greatly contribute to a wide variety of medical applications as well as maintenance of health in humans. Here, our attempts to understand seasonally controlled hibernation as a physiological system for maintaining health and life are reviewed.

\section{SEASONALLY REGULATED TOLERANCE OF HEARTS TO LOW Tb}

As regulated heart function is the most important factor for maintaining life, there has been great interest in the physiological characteristics of hearts in a hibernating state that bestow tolerance to a remarkably decreased $\mathrm{Tb}$ (Wang 1988). The first evidence that the source of calcium ions $\left(\mathrm{Ca}^{2+}\right)$ for cardiac excitation-contraction coupling is modulated during hibernation was demonstrated by an electrophysiological and pharmacological study of the hearts of chipmunks (Tamias sibiricus), a rodent hibernator (Kondo and Shibata 1984). In cardiac muscles from hibernating chipmunks, the plateau phase of a membrane action potential, which is generated by $\mathrm{Ca}^{2+}$ currents carried by trans-sarcolemmal $\mathrm{Ca}^{2+}$ influx through $\mathrm{Ca}^{2+}$ channels, is markedly inhibited. This inhibited plateau phase is attributed to a remarkably decreased activation of voltage-dependent $\mathrm{Ca}^{2+}$ channels (Kondo 1986a) by a rapid repolarization below their activation threshold by enhanced transient $\mathrm{K}^{+}$outward currents that are sensitive to 4-aminopyridine (Kondo 1986b). However, despite much less $\mathrm{Ca}^{2+}$ influx through $\mathrm{Ca}^{2+}$ channels, the contraction was substantially unchanged between active and hibernating animal myocardium. The discrepancy between decreased trans-sarcolemmal $\mathrm{Ca}^{2+}$ inflow and unchanged contraction was solved by experiments using ryanodine and caffeine, which are inhibitors of $\mathrm{Ca}^{2+}$ release and uptake, respectively, by the sarcoplasmic reticulum (SR), which are internal $\mathrm{Ca}^{2+}$ store sites; the ability of the SR to take up and release $\mathrm{Ca}^{2+}$ is greatly augmented in myocardium from hibernating animals (Kondo 1988). It is believed that this augmentation compensates for decreased $\mathrm{Ca}^{2+}$ inflow through $\mathrm{Ca}^{2+}$ channels for maintaining homeostasis of cardiac function. Such an augmented ability of the SR is supported by a study on cardiac SR vesicles from hibernating ground 
squirrels which found that both the rate of $\mathrm{Ca}^{2+}$ uptake and level of $\mathrm{Ca}^{2+}$ accumulation are increased (Belke et al. 1991), as well as a recent study that reported the gene expression level of the SR $\mathrm{Ca}^{2+}$-ATPase but not $\mathrm{Ca}^{2+}$ binding protein calsequestrin is increased in hibernating woodchuck (Yatani et al. 2004).

The results of these studies suggest that in hibernating animals, intracellular $\mathrm{Ca}^{2+}$ homeostasis for cardiac contraction and relaxation is maintained primarily by internal $\mathrm{Ca}^{2+}$ release and reuptake by the modulated SR (Fig. 1, top) (Kondo 1997). This modulation would maintain regulated functions of the heart at low $\mathrm{Tb}$ and simultaneously greatly economize on energy that is consumed to remove cytosolic $\mathrm{Ca}^{2+}$ for relaxation because an excess amount of $\mathrm{Ca}^{2+}$ is not loaded in cells due to the supply of a moderate amount of $\mathrm{Ca}^{2+}$ from the SR that stores the limited $\mathrm{Ca}^{2+}$. Thus, the modulation of $\mathrm{Ca}^{2+}$ sources in myocardium from hibernating animals establishes an ideal intracellular $\mathrm{Ca}^{2+}$ recycling system for the prevention of cellular $\mathrm{Ca}^{2+}$ overload by enhancing the SR ability and avoiding excess $\mathrm{Ca}^{2+}$ influx induced by an extensively delayed inactivation process of $\mathrm{Ca}^{2+}$ channels at low $\mathrm{Tb}$. The switching to this recycling system would increase the capacity of hearts for tolerating low Tb. A similar cardiac modulation has been observed in two other hibernators, ground squirrels and woodchucks (Wang et al. 2002; Yatani et al. 2004), suggesting a common mechanism for the prevention of cold damage in mammalian hibernators.

The modulation of cardiac $\mathrm{Ca}^{2+}$ regulation found in hibernating chipmunks is observed even in normothermic chipmunks in which a decreased $\mathrm{Tb}$ is prevented throughout a year by keeping them under conditions of constant warmth $\left(23^{\circ} \mathrm{C}\right)$ and a 12-hour light/dark cycle (Kondo 1987; Kondo and Kondo 1992b). The cardiac electromechanical properties are switched from extracellular $\mathrm{Ca}^{2+}$ dependent properties to the $\mathrm{SR} \mathrm{Ca}^{2+}$-dependent properties only in the winter season under constant laboratory conditions (Fig. 1, bottom). This result clearly indicates that switching to unique properties seen in hibernating animals seasonally occurs even in normothermic animals, suggesting that the hibernation-like modulation is seasonally caused independently of $\mathrm{Tb}$ and environmental changes and completed before the onset of hibernation for tolerating low Tb. From these findings, it can be surmised that a seasonal timing mechanism functions endogenously in organisms and systemically controls the ability to adapt to severe physiological states during the hibernation season. To understand the mechanism by which hibernation is controlled, a seasonally regulated molecular probe specific for hibernation, which should be correlated with the seasonal modulation in hearts, has been explored.

\section{HIBERNATION MARKER AND CIRCANNUAL HIBERNATION RHYTHM}

In the blood of chipmunks, liver-derived protein complex has been isolated as a factor that is correlated with seasonally changed electromechanical properties in cardiac muscles (Kondo and Kondo 1992b). This complex is composed of four novel proteins termed hibernation-spe-
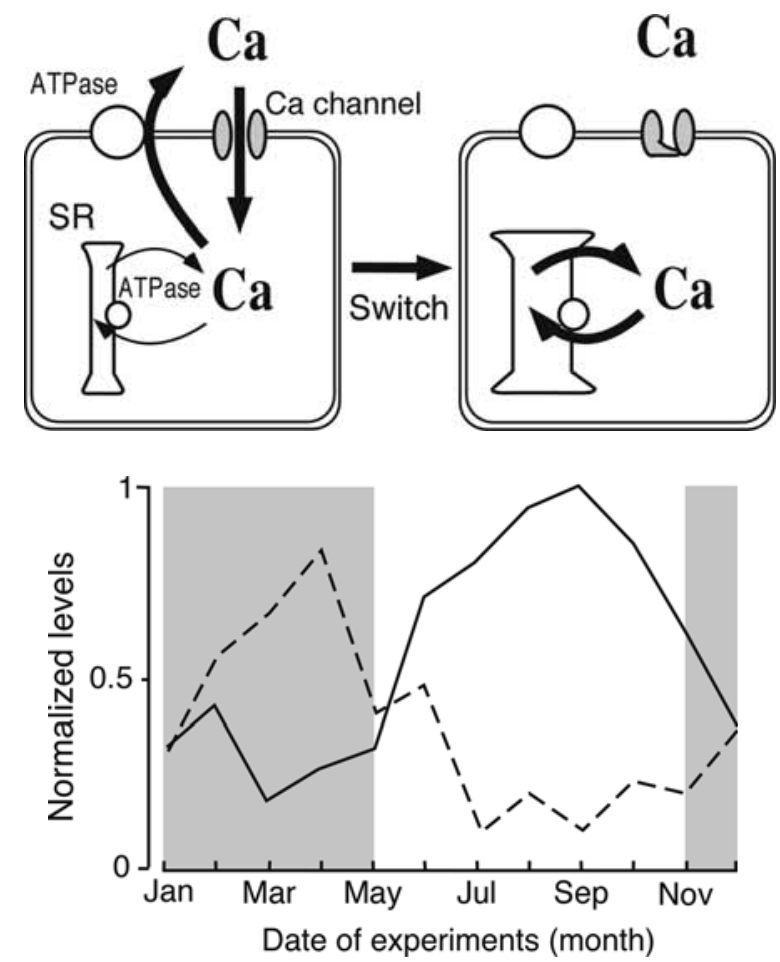

Figure 1. Modulation of $\mathrm{Ca}^{2+}$ source for cardiac excitation-contraction coupling during hibernation. (Top) Modulation is circannually switched from active (left) to hibernating (right) state. (Bottom) Circannual changes in electromechanical properties dependent on trans-sarcolemmal $\mathrm{Ca}^{2+}$ influx (solid line) and the $\mathrm{SR} \mathrm{Ca}^{2+}$ release (dashed line) occur in animals kept under conditions of constant warmth and a 12-hour light/dark cycle. (Gray area) Period of hibernation-like changes; (vertical axis) normalized levels of dependence with respect to differences in the degree of dependence between active and hibernating animals myocardium.

cific proteins (HP) (Kondo and Kondo 1992a). Three of four HPs (HP20, HP25, and HP27) have homologous structures to a collagen-like domain and form a heterotrimer complex (HP20c) through a triple-helix structure in amino-terminal regions (Fig. 2). HP20c further associates with the fourth protein, HP55, which is a pro-

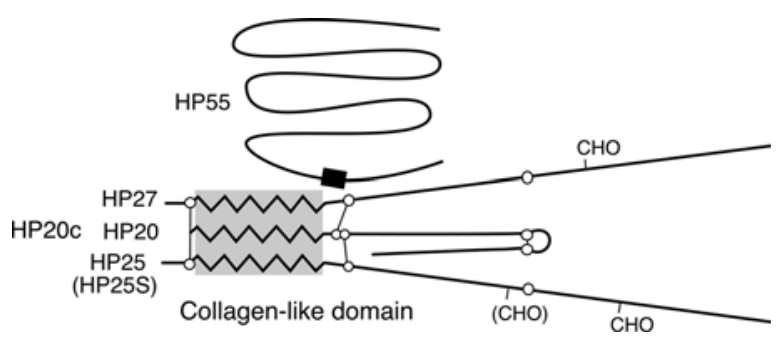

Figure 2. Characteristics of the HP complex structure. The lengths of the lines correspond to the number of amino acids. Gray area in HP20c indicates a collagenous domain forming a triple helix. Open circles and CHOs indicate cysteine residues and glycosylation sites, respectively. Lines between open circles show disulfide bonds. HP25S is a glycosylated HP25. Closed box in HP55 shows an active center and it is assumed it interacts with HP20c at this position. 


\section{CIRCANNUAL HIBERNATION CLOCK AND HP COMPLEX}

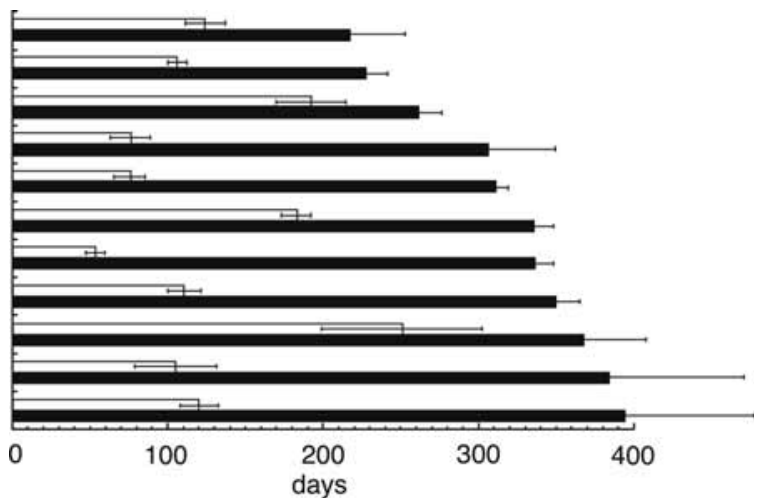

Figure 3. Circannual hibernation rhythms. Cycle periods (closed bars) and durations (open bars) of free-running hibernation in 11 animals maintained under conditions of constant cold and darkness. Lines attached to bars show mean \pm S.E.M. $(n=$ between 5 and 9).

tease inhibitor in the serpin superfamily (Kondo and Kondo 1996), in the blood by a hydrophobic bond (Kondo and Kondo 1992a). HP complex (complex of HP20c with HP55) in the blood is down-regulated in correlation with hibernation by depressed expression of genes in the liver (Kondo and Kondo 1992a; Takamatsu et al. 1993). On the basis of the structural characteristics of the HP complex, it seems likely that HP20c is inactivated by binding to HP55 in the blood (Kondo and Kondo 1992a).

To clarify the correlation between the HP complex and hibernation, the rhythmicity of hibernation in chipmunks has been examined throughout their lives under constant laboratory conditions. Under conditions of constant cold $\left(5^{\circ} \mathrm{C}\right)$ and 24 -hour darkness, the animals exhibited a freerunning hibernation rhythm with each individual circannual timing (Fig. 3). The circannual cycle and the duration of hibernation range between 5 and 13 months and between 2 and 8 months, respectively, and are relatively constant in individuals. There is no correlation between the cycle period and the duration. Surprisingly, the maximum life span of these animals with a circannual hibernation rhythm was approximately 11 years, which is four times longer than rats and mice (Kondo et al. 2006). However, a few animals that were unable to hibernate throughout their life had only short life spans (up to 3 years). Thus, the timing of hibernation is controlled by a system generating endogenous circannual rhythm characterized in each individual. Animals that undergo hibernation have an exceptionally long life span.

The down-regulation of the HP complex is closely correlated with a circannual hibernation rhythm (Kondo et al. 2006); levels of HP complex in the blood are decreased prior to the onset of hibernation and kept low during hibernation, following which the decreased levels return to active-state levels in association with the termination of hibernation (Fig. 4). Such a down-regulation of the HP complex in the blood was due to depressed expression of HP genes in the liver. The fact that down-regulation of the HP complex is turned on and off before the onset and termination of hibernation, respectively, implies that a circannual timing mechanism for hibernation controls expression of the HP complex. Interestingly, in a few animals that are unable to hibernate under conditions of constant cold and darkness, the HP complex in the blood is never down-regulated, suggesting an important role for the HP complex in hibernation. This is supported by the species-specific expression of HP genes in hibernators; HP genes are expressed in hibernators, such as ground squirrels and woodchucks (Kondo and Kondo 1993), whereas in tree squirrels, which are nonhibernators of the same squirrel family as chipmunks, HP genes are not expressed due to a base substitution in the promoter region (Kojima et al. 2001).

More significant evidence for a circannual regulation of the HP complex and its role in hibernation has been shown in animals in which a decreased $\mathrm{Tb}$ was prevented by maintaining them under conditions of constant warmth $\left(23^{\circ} \mathrm{C}\right)$ and a 12-hour light/dark cycle (Kondo et al. 2006). Even in such normothermic animals, the HP complex in the blood is circannually regulated, and the timing and duration of down-regulation of the HP complex are similar to those in circannually hibernating animals kept under conditions of constant cold and darkness, demonstrating that a circannual timing mechanism functions independently of Tb and environment changes. Only when the level of the HP complex in the blood is down-regulated do animals develop decreased $\mathrm{Tb}$ and begin hibernation due to exposure to cold. This indicates that a circannually regulated decrease in the HP complex in the blood is necessary for hibernation; during this decrease, the ability to tolerate low $\mathrm{Tb}$ may be developed in order to survive the hypothermic state. The similarities in the timing and duration of circannual HP down-regulation between animals kept in constant warm and cold environments imply that an endogenous circannual clock system that generates a circannual rhythm is insensitive to $\mathrm{Tb}$ and environmental changes.

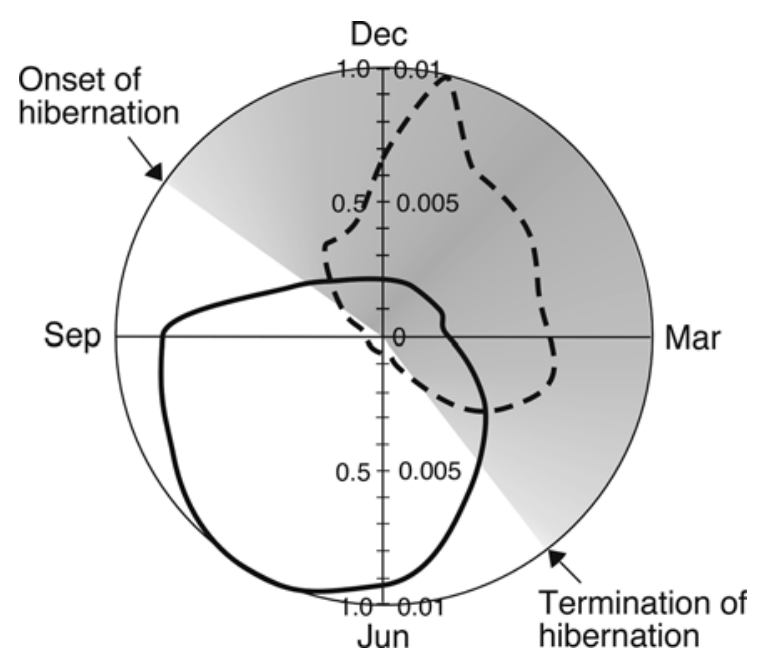

Figure 4. Link of circannually regulated HP complex in the blood and CSF to hibernation. A cycle of the HP complex and hibernation rhythm in animals kept under conditions of constant cold and darkness was fitted to a year. (Solid lines) HP levels in the blood; (dashed lines) HP levels in the CSF. (Numbers on vertical axis) HP levels in the blood (left) and CSF (right) normalized to their maximum content in the blood. 


\section{INCREASED HP COMPLEX IN THE BRAIN DURING HIBERNATION}

By immunochemical analysis of cerebrospinal fluid (CSF) using antibodies against components of the HP complex, it has been demonstrated that the HP complex is markedly increased in CSF during hibernation despite its decrease in the blood (Fig. 4) (Kondo et al. 2006). An increase in the HP complex in CSF during hibernation is due to circannually up-regulated transcytosis of the HP complex through the choroidal epithelium (the bloodCSF barrier) but not to the HP gene expression in the brain. As levels of the HP complex in the blood are higher than those in the CSF even during hibernation, a sufficient amount of the HP complex exists in the blood for supplying to the CSF. The up-regulation of the HP complex in CSF is coincident with a circannual down-regulation of that in the blood (Fig. 4), and similar regulation in CSF and blood is observed in normothermic animals in which a decreased $\mathrm{Tb}$ was prevented by keeping them under conditions of constant warmth and a 12-hour light/dark cycle, indicating that a circannual timing mechanism simultaneously and conversely regulates the HP complex in the blood and CSF independently of $\mathrm{Tb}$ and environmental changes. This suggests that a circannual clock outputs a signal simultaneously to the liver for decreasing HP production and to the choroidal epithelium for increasing HP transport. Thus, the HP complex is increased in the brain under control of an endogenous circannual rhythm and then hibernation begins.

Circannually facilitated HP transport through the blood-CSF barrier may have a critical role in the functioning of the HP complex in the brain because the HP complex dissociates to HP20c and HP55 in CSF (Kondo et al. 2006). Because the HP complex exists in the blood as a complex consisting of an interaction between HP20c and HP55, which is probably an inactive form (Kondo and Kondo 1992a), HP20c that has dissociated from HP55 is likely to be an active form. Such an activation of the HP complex through the blood-CSF barrier may be controlled by a signal outputted by a circannual clock. This implies that the brain is a target of HP20c for control of hibernation.

\section{THE HP COMPLEX ESSENTIAL FOR HIBERNATION}

Studies on the effects of administration of antibody against HP20c into lateral ventricles on hibernation have demonstrated the essential role of circannually increased brain HP20c in hibernation (Kondo et al. 2006). The administered antibody decreases the amount of time spent in low $\mathrm{Tb}$ (hibernation time) in a dose-dependent manner. In some animals, hibernation was interrupted during antibody administration, whereas in other animals, antibody administered in the late stage of hibernation accelerated the termination of hibernation. Thus, blocking HP20c activity in the brain with antibody dramatically decreases hibernation time.

In this study, the concept of "hibernation time" may be primarily used for the quantitative evaluation of hiberna- tion (Kondo et al. 2006). During hibernation, animals periodically arouse for several hours after spending several days at a continuous low $\mathrm{Tb}$. In chipmunks, periods of this periodic arousal and continuous low Tb are relatively constant in each individual, and periodic arousal is necessary for surviving low $\mathrm{Tb}$ during hibernation bouts. As low $\mathrm{Tb}$ is potentially lethal in cells due to the metabolic imbalance ascribed to perturbing cellular ion regulation and energy metabolism that is accumulated over time, periodic arousal may be essential for reestablishing the metabolic balance for surviving (Wang 1989). If this periodic arousal is disturbed, the animal would die. Therefore, time spent in continuous low $\mathrm{Tb}$ during hibernation bouts means the limit of time survived at a decreased $\mathrm{Tb}$. This limit of time may correspond to a capacity for tolerating low $\mathrm{Tb}$. Even nonhibernators, including humans, can tolerate low $\mathrm{Tb}$, although the length of time survived at low $\mathrm{Tb}$ is much less than that in hibernators. For example, humans can survive less than 1 hour at $\mathrm{Tb}$ near $20^{\circ} \mathrm{C}$, whereas chipmunks survive approximately 7 days ( 168 hours) below $10^{\circ} \mathrm{C}$ during the hibernation season. Such a prolonged time may depend on the capacity for tolerating low Tb developed by a circannually regulated ability to hibernate. The fact that antiHP20c antibody administered into the brain dramatically decreases hibernation time or interrupts hibernation suggests that brain HP20c is essential for developing a capacity for protecting organisms not only from low $\mathrm{Tb}$, but also from other lethal factors and events during hibernation.

\section{PROPOSED ROLE OF THE HP COMPLEX}

Although the exact function of the HP complex in the brain and peripheral organs remains to be elucidated, some significant roles for HP20c have been proposed based on the results of studies on members of HP20c family proteins, which have structural and evolutional similarities to HP20c, such as complement protein C1q, precerebellin, ACRP30/adiponectin, and tumor necrosis factor (TNF) family (Kishore et al. 2004). The liver-derived C1q is involved in activation of the classical complement pathway responsible for antimicrobial defense and immunological processes such as immune tolerance, phagocytosis of bacteria, neutralization of retroviruses, and modulation of dendritic cells, B cells, and fibroblasts. Precerebellin is expressed in the cerebellum and may have a role in the development and stability of Purkinje cell synapses. Adiponectin derived from adipocytes is known to regulate lipid and carbohydrate catabolism for maintaining energy homeostasis and resolve inflammation. The TNF family members are involved in adaptive immunity, apoptosis selectively in tumor cells, inflammation, cell survival and proliferation, and bone and energy homeostasis. Thus, proteins that are structurally homologous to HP20c have been shown to contribute to energy homeostasis, immunity, tumor cell apoptosis, and cell survival. Because similar biological effects for HP20c have been proposed, an increase in brain HP20c during hibernation may protect organisms from depressed metabolism and lethal diseases as observed during hibernation in hibernators. An essential role for brain HP20c in tolerating low $\mathrm{Tb}$ (Kondo et al. 2006) is compatible with this possibility. 


\section{CIRCANNUAL HIBERNATION CLOCK AND HP COMPLEX}

\section{CIRCANNUAL HP RHYTHM FOR MAINTAINING LIFE}

Studies of the hearts of hibernators have suggested that circannually modulated cardiac $\mathrm{Ca}^{2+}$ regulation independent of $\mathrm{Tb}$ and environmental changes develops cardiac tolerance to lethal low $\mathrm{Tb}$ by preventing cellular $\mathrm{Ca}^{2+}$ overload. This may be expanded into the protection of principal organs and tissues from low $\mathrm{Tb}$ because a $\mathrm{Ca}^{2+}$ overloaded state causes lethal damage in various cells by activation of $\mathrm{Ca}^{2+}$-dependent necrotic processes such as proteolysis, lipid peroxidation, and mitochondrial swelling. Therefore, a circannual timing mechanism may have a central role in systemic modulation of cellular functions for protecting organisms from lowering of $\mathrm{Tb}$ via a signaling pathway. The HP complex is believed to be the first promising candidate hormone that carries a circannual signal into the brain for protecting organisms, most likely by maintaining cellular ion balance, energy homeostasis, and immune functions.

Our preliminary study of the longevity of chipmunks (Kondo and Narita 1998; Kondo 1999) led to the surprising involvement of circannually controlled HP complex in maintaining health and life. Animals with a circannual HP rhythm that undergo lowering of $\mathrm{Tb}$ during hibernation seasons by being kept under conditions of constant cold or those that maintain a normothermic state throughout by being kept under conditions of constant warmth have a much longer life span; many of these animals live close to 10 years and the maximum life span is over 10 years, which is approximately four or five times longer than rats and mice. On the other hand, animals without circannual HP rhythm have a short life span of only a few years, similar to rodent nonhibernators. This result suggests that animals in which brain HP20c is circannually increased live much longer and in good health, probably due to the prevention of lethal diseases and slowing of aging. Because such a long life span is not due to depressed metabolism by low $\mathrm{Tb}$ during hibernation, it is further suggested that hibernation should be redefined as the ability to govern modulation of cellular functions for maintaining a long life through hormonal signaling, rather than the ability to decrease $\mathrm{Tb}$. Namely, mammalian hibernation may be controlled by a physiological system consisting of a circannual oscillator, its signaling pathway, and target sites. The HP complex may be an essential modulator for governing cellular tolerance to metabolic depression, diseases, and aging.

\section{CIRCANNUAL RHYTHM AND CIRCADIAN RHYTHM}

A circannual rhythm has a central role in the survival of homeothermic animals in nature; this rhythm is responsible for controlling the timing of reproduction in mammals and migration in birds, which are critical for survival. However, there are serious problems with respect to studying circannual timing mechanisms because of the long cycle of a circannual rhythm, influences of environmental changes such as light and temperature, and its expression at the organism level. By identifying the HP complex as a unique marker of a circannual rhythm inde- pendent of $\mathrm{Tb}$ and environmental changes, a circannual timing mechanism has been characterized without any influence by environmental and $\mathrm{Tb}$ changes.

Because HP rhythm is correlated with a circannual hibernation rhythm, the influences of Tb and environmental changes on a circannual rhythm can be explored by comparing animals subjected to a decreased $\mathrm{Tb}$ under conditions of constant cold $\left(5^{\circ} \mathrm{C}\right)$ and darkness and normothermic animals under conditions of constant warmth $\left(23^{\circ} \mathrm{C}\right)$ and a 12-hour light/dark cycle (Kondo et al. 2006). A circannual HP rhythm is generated independently of Tb change, indicating that lowering of $\mathrm{Tb}$ does not trigger the timing of the rhythm. Furthermore, cycle periods and duration of hibernation in animals maintained under cold conditions are similar to those of HP down-regulation in normothermic animals kept under constant warm conditions, indicating that the generation of circannual rhythms is not affected by differences in $\mathrm{Tb}$, environmental temperature, and light between animals kept under cold and warm conditions. Thus, a circannual clock mechanism maintains homeostasis even at $\mathrm{Tb}$ near $0^{\circ} \mathrm{C}$ and is little affected by light.

Circadian rhythms are known to possess temperature compensation properties that are similar to those of circannual HP rhythms, and it has been proposed that seasonal rhythms depend on the circadian system. As circadian rhythms are generated by negative feedback loops that are formed by core circadian clock genes and their products, this or similar mechanisms are assumed to function in the generation of a circannual rhythm. However, although free-running circadian periods are longer than 24 hours and can be reset by light, cycle periods of free-running circannual rhythms are shorter than 1 year and unaffected by the difference between darkness and a 12-hour light/dark cycle. A circadian rhythm can be generated in a variety of organs and tissues in organisms and even in monocytes, whereas a circannual rhythm would be generated only at the organism level. A circannual rhythm in chipmunks is insensitive to aging (Kondo et al. 2006), whereas a circadian rhythm in rodents is affected by aging (Bentivoglio et al. 2006). The suggested direct link between circadian cycles and metabolic cycles (Rutter et al. 2002) may be incompatible with the result that a free-running circannual cycle of the HP complex is not affected by a decreased $\mathrm{Tb}$ during hibernation that dramatically depressed metabolic processes. These characteristics suggest a lesser degree of similarity between circannual and circadian timing mechanisms. Therefore, a circannual rhythm may not be generated by estimating time on the basis of circadian rhythms but could be modulated by circadian rhythms for adapting periods of freerunning endogenous circannual rhythms to a seasonal cycle in nature. Systemic rather than cellular feedback loops may be involved in circannual rhythm generation because of the insensitivity of this rhythm to $\mathrm{Tb}$ changes and its systemic expression at the organism level. Our proposed cross-talk model for a hibernation control system (Kondo et al. 2006) implies the involvement of a systemic negative feedback loop formed by the HP complex and a signaling pathway between the brain and periphery in the generation of a circannual rhythm (Fig. 5). 


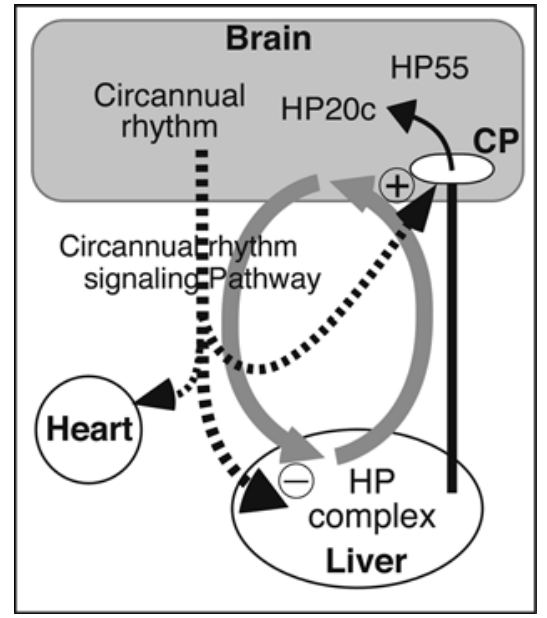

Figure 5. Proposed system for systemic circannual rhythm generation. Inverse regulation of HP complex in the brain (+: upregulation) and liver (-: down-regulation) may systemically form a negative feedback loop between the brain and periphery (gray arrow). (Dashed arrow) Predicted circannual rhythm signaling pathway from the brain to periphery. (CP) Choroid plexus (blood-CSF barrier).

\section{HIBERNATION AND SLEEP}

Although similarities between hibernation and sleep have been suggested by a decreased Tb during either sleep or hibernation and reports that entry into hibernation is an extension of slow-wave sleep (Berger 1984; Krilowicz et al. 1988), there may be serious differences between them as shown below. The amplitude of decreased $\mathrm{Tb}$ during sleep is less than $1{ }^{\circ} \mathrm{C}$, whereas that during hibernation is more than $30^{\circ} \mathrm{C}$, and sleep does not develop a capacity for tolerating low $\mathrm{Tb}$, although hibernation does. Hibernation cycles are controlled by a circannual rhythm, and circadian rhythms may have a role in sleep homeostasis. Although the amount of time spent in sleep is in proportion to that spent in wakefulness, there is no correlation between periods of hibernation and active state. Furthermore, an analysis of electroencephalograms suggested that slow-wave sleep as restoration from wakefulness is disturbed during hibernation (Daan et al. 1991). Thus, the mechanism(s) of hibernation may not be based on sleep.

Our recent study on the role of the HP complex in hibernation (Kondo et al. 2006) demonstrated that a sleeplike inactive state induced by a decreased $\mathrm{Tb}$ is not necessary for the ability to hibernate because the ability to survive a state of hibernation is circannually developed independently of decreased Tb. Furthermore, the fact that chipmunks exhibiting circannual HP rhythm have a surprisingly long life span with or without $\mathrm{Tb}$ lowering reveals that an inactive state due to a low Tb during hibernation is not essential, although sleep is, for the maintenance of life.

The above discussion indicates that an inactive state during hibernation is not of primary importance for life processes, although sleep is critical and inevitable for these processes, and that in a hibernating state but not sleep, organisms maintain regulated function at the molecular, cellular, and organ levels at a potentially lethal low $\mathrm{Tb}$. This implies that there is a minimal degree of similarity between the mechanisms of hibernation and sleep.

\section{CONCLUSIONS}

A circannual rhythm responsible for hibernation controls the modulation of $\mathrm{Ca}^{2+}$ sources for cardiac excitation-contraction coupling independently of $\mathrm{Tb}$ and environmental changes, and this rhythm establishes an intracellular $\mathrm{Ca}^{2+}$ recycling system for the prevention of cellular $\mathrm{Ca}^{2+}$ overload at low Tb. This modulation may lead to the development of a capacity for hearts to tolerate low $\mathrm{Tb}$ during the hibernation season. The liver-derived HP complex was identified as being a novel hormone essential for hibernation in the brain. Under control of an endogenous circannual rhythm, the HP complex is downregulated in the blood and concurrently up-regulated in CSF through active transport via the blood-CSF barrier. The HP complex is activated in the brain for developing a capacity for tolerating a severely depressed metabolic state by low $\mathrm{Tb}$ and would help the animal live longer. These results suggest that a circannually regulated physiological system that controls hibernation via the HP complex maintains health and life and allow us to discuss circannual rhythms versus circadian rhythms and hibernation versus sleep. Simultaneous and inverse regulation of the HP complex in the blood and the brain led us to propose that a circannual rhythm is generated by a systemic negative feedback loop formed between the brain and periphery.

\section{ACKNOWLEDGMENTS}

This work was supported by the Kanagawa Academy of Science and Technology and the National Space Development Agency of Japan. I thank all of the researchers and research assistants for their valuable collaboration during this project.

\section{REFERENCES}

Belke D.D., Milner R.E., and Wang L.C.H. 1991. Seasonal variations in the rate and capacity of cardiac SR calcium accumulation in a hibernating species. Cryobiology. 28: 354.

Bentivoglio M., Deng X.H., Nygard M., Sadki A., and Kristensson K. 2006. The aging suprachiasmatic nucleus and cytokines: Functional, molecular, and cellular changes in rodents. Chronobiol. Int. 23: 437.

Berger R.J. 1984. Slow wave sleep, shallow torpor and hibernation: Homologous states of diminished metabolism and body temperature. Biol. Psychol. 19: 305.

Daan S., Barnes B.M., and Strijkstra A.M. 1991. Warming up for sleep?: Ground squirrels sleep during arousals from hibernation. Neurosci. Lett. 128: 265.

Donahue S.W., Galley S.A., Vaughan M.R., PattersonBuckendahl P., Demers L.M., Vance J.L., and McGee M.E. 2006. Parathyroid hormone may maintain bone formation in hibernating black bears (Ursus americanus) to prevent disuse osteoporosis. J. Exp. Biol. 209: 1630.

Kishore U., Gaboriaud C., Waters P., Shrive A.K., Greenhough T.J., Reid K.B.M., Sim R.B., and Arlaud G.J. 2004. C1q and tumor necrosis factor superfamily: Modularity and versatility. Trends Immunol. 25: 551. 


\section{CIRCANNUAL HIBERNATION CLOCK AND HP COMPLEX}

Kojima M., Shiba T., Kondo N., and Takamatsu N. 2001. The tree squirrel HP-25 gene is a pseudogene. Eur. J. Biochem. 268: 5997.

Kondo J. and Kondo N. 1996. Structural aspects of complex of hibernation-specific proteins. In Adaptation to the cold: Tenth International Hibernation Symposium (ed. F. Geiser et al.), p. 351. University of New England Press, Armidale, Australia.

Kondo N. 1986a. Excitation-contraction coupling in myocardium of nonhibernating and hibernating chipmunks: Effects of isoprenaline, a high calcium medium and ryanodine. Circ. Res. 59: 221.

1986b Excitation-contraction coupling in the myocardium of hibernating chipmunks. Experientia 42: 1220. . 1987. Identification of a pre-hibernating state in myocardium from nonhibernating chipmunks. Experientia 43: 873.

.1988. Comparison between effects of caffeine and ryanodine on electromechanical coupling in myocardium of hibernating chipmunks: Role of internal $\mathrm{Ca}$ stores. $\mathrm{Br} . J$. Pharmacol. 95: 1287.

- 1997. Physiological and biochemical studies on hibernation control mechanism in mammalian hibernation. In Sleep and sleep disorders: From molecule to behavior (ed. O. Hayaishi and S. Inoue), p. 129. Academic Press, Tokyo, Japan. 1999. Hibernation control mechanism and possible applications to humans. J. Br. Interplanet. Soc. 58: 343.

Kondo N. and Kondo J. 1992a. Identification of novel blood proteins specific for mammalian hibernation. J. Biol. Chem. 267: 473.

. 1992b. Identification of novel types of protein specific for mammalian hibernation with circannual rhythm. In Circadian clocks from cell to human (ed. T. Hiroshige and K. Honma), p. 89. Hokkaido University Press, Sapporo, Japan. . 1993. Identification and characterization of novel types of plasma protein specific for hibernation in rodents. In Life in the cold: Ecological, physiological and molecular mechanisms (ed. C. Carey et al.), p. 467. Westview Press, Boulder, Colorado.

Kondo N. and Narita A. 1998. Does hibernation increase survival rate in chipmunks? Zoolog. Sci. (suppl.) 15: 106.

Kondo N. and Shibata S. 1984. Calcium source for excitationcontraction coupling in myocardium of nonhibernating and hibernating chipmunks. Science 225: 641.

Kondo N., Sekijima T., Kondo J., Takamatsu N., Tohya K., and Ohtsu T. 2006. Circannual control of hibernation by HP complex in the brain. Cell 125: 161.

Krilowicz B.L., Glotzbach S.F., and Heller H.C. 1988. Neuronal activity during complete bouts of hibernation. Am. J. Physiol. 255: R1008.

Maniero G.D. 2002. Classical pathway serum complement activity throughout various stages of the annual cycle of a mammalian hibernator, the golden-mantled ground squirrel, Spermophilus lateralis. Dev. Comp. Immunol. 26: 563.

2005. Ground squirrel splenic macrophages bind lipopolysaccharide over a wide range of temperatures at all phases of their annual hibernation cycle. Comp. Immunol. Microbiol. Infect. Dis. 28: 297.

Rutter J., Reick M., and McKnight S.L. 2002. Metabolism and the control of circadian rhythms. Annu. Rev. Biochem. 71: 307.

Takamatsu N., Ohba K.-I., Kondo J., Kondo N., and Shiba T. 1993. Hibernation-associated gene regulation of plasma proteins with a collagen-like domain in mammalian hibernators. Mol. Cell. Biol. 13: 1516.

Wang L.C.H. 1988. Mammalian hibernation: An escape from the cold. In Advances in comparative and environmental physiology (ed. R. Gilles), vol. 2, p. 1. Springer-Verlag, Berlin.

1989. Ecological, physiological, and biochemical aspects of torpor in mammals and birds. In Advances in comparative and environmental physiology (ed. R. Gilles), vol. 4, p. 361. Springer-Verlag, Berlin.

Wang S.Q., Lakatta E.G., Cheng H., and Zhou Z.Q. 2002. Adaptive mechanisms of intracellular calcium homeostasis in mammalian hibernators. J. Exp. Biol. 205: 2957.

Yatani A., Kim S.J., Kudej R.K., Wang Q., Depre C., Irie K., Kranias E.G., Vatner S.F., and Vatner D.E. 2004. Insights into cardioprotection obtained from study of cellular $\mathrm{Ca}^{2+}$ handling in myocardium of true hibernating mammals. Am. J. Physiol. Heart Circ. Physiol. 286: H2219.

Zivadinovic D. and Andjus R.K. 1996. Life span of the European ground squirrel Spermophilus citellus under free-running conditions and entrainment. In Adaptations to the cold: Tenth International Hibernation Symposium (ed. F. Geiser et al.), p. 103. University of New England Press, Armidale, Australia. 


\section{$\overbrace{\text { CSH' }}^{\infty}$ Cold Spring Harbor Symposia SYMPOSIA on Quantitative Biology}

\section{Endogenous Circannual Clock and HP Complex in a Hibernation Control System}

N. Kondo

Cold Spring Harb Symp Quant Biol 2007 72: 607-613

Access the most recent version at doi:10.1101/sqb.2007.72.028

References This article cites 25 articles, 6 of which can be accessed free at:

http://symposium.cshlp.org/content/72/607.full.html\#ref-list-1

License

Email Alerting Receive free email alerts when new articles cite this article - sign up in the box at the Service top right corner of the article or click here. 